\title{
INTERACTIONS DUE TO MOVING HEAT SOURCES IN GENERALIZED THERMOELASTIC HALF-SPACE USING L-S MODEL
}

\author{
N. SARKAR \\ Department of Mathematics, Jadavpur University \\ Kolkata-700032, INDIA \\ E-mail: nantu.math@gmail.com \\ A. LAHIRI* \\ Department of Mathematics, Jadavpur University \\ Kolkata-700032, INDIA \\ E-mail: lahiriabhijit2000@yahoo.com
}

\begin{abstract}
A one-dimensional problem for a homogeneous, isotropic and thermoelastic half-space subjected to a moving plane of heat source on the boundary of the space, which is traction free, is considered in the context of LordShulaman model (L-S model) of thermoelasticity. The Laplace transform and eigenvalue approach techniques are used to solve the resulting non-dimensional coupled equations. Numerical results for the temperature, thermal stress, and displacement distributions are represented graphically and discussed.
\end{abstract}

Key words: generalized thrmoelasticity, L-S model, elastic half-space, moving heat sources, eigenvalue approach.

\section{Introduction}

The coupling between thermal and strain fields gives rise to the coupled theory of thermoelasticity (CTE). During last few decades, the generalized theories of thermoelasticity involving finite speed of heat transportation in an elastic solid have been the center of active research to remove the paradox of infinite speed of heat propagation inherent in the dynamical coupled theory of thermoelasticity (CTE) developed by Biot (1956). The governing equations involved in these theories are of hyperbolic type because of the introduction of thermal relaxation time parameters. To eliminate these type of a shortcoming, various modified generalized thermoelasticity theories were proposed by Lord and Shulman (1967) (LS model), Green and Lindsay (1972) (GL model) and Green and Naghdi (1991; 1992; 1993) (GN models) based on "second sound" effects, i.e., propagation of heat as a wave like phenomenon.

In 1967, Lord and Shulman attempt to eliminate the paradox of infinite velocity of thermal disturbances inherent in the CTE. This model is based on a modified Fourier's law but in addition a single relaxation time was considered. This theory was extended by Dhaliwal and Sherief (1980a) to include the anisotropic case. The uniqueness of the solution for this theory was proved under different conditions by Ignaczak (1979; 1982), by Dhaliwal and Sherief (1980b) and by Sherief (1987). In the L-S model, finite speed of thermal disturbance has been considered with thermal relaxation time. The heat conduction equation in this model is of hyperbolic type and is closely connected with the theories of second sound.

Green and Lindsay (1972) also proposed a theory of generalized thermoelasticity with two relaxation time parameters and modified both the energy equation and constitutive equations. The GL model admits second sound without violating Fourier's law. Both the theories are structurally different, but one can be obtained as a particular case of the other. Various problems related to the above theories have been

\footnotetext{
${ }^{*}$ To whom correspondence should be addressed
} 
investigated by Youssef (2006; 2009), Sherief and Megahed (1999), Sherief and Youssef (2004), Ezzat et al. (2001), Lahiri et al. (2010a; 2010b).

A recent theoretical development of this subject is due to Green and Naghdi $(1991 ; 1992 ; 1993)$. Providing sufficient basic modifications in the governing equations Green and Naghdi developed a new general theory of thermoelasticity that permits treatment of a much wider class of heat flow problems. They divided their theory into three parts and referred as types I, II and III. The linearized version of constitutive equations of GN I (1991) is same as the classical thermoelasticity theory which is based on Fourier's law, whereas the linearized version of GN II (1993) theory permits propagation of thermal waves at finite speed. In the GN-II model, the internal rate of production of entropy is assumed to be identical to zero, i.e., there is no dissipation of thermal energy and this theory is referred to as thermoelasticity without energy dissipation theory. In development of the GN III (1992) model, the constitutive equations are derived by including a thermal displacement gradient in addition to a temperature gradient among the constitutive variables. However, this model admits dissipation of energy in general.

Most of the thermoelasticity (generalized or coupled) problems have been solved by using the potential function. This method is not always suitable as discussed by Anwar and Sherief (1988) and Sherief (1993). Their discussion may be summarized by (I) the boundary and initial conditions for physical problems are directly related to the physical quantities under consideration and not to the potential function and (II) the solution of the physical problem in natural variables is convergent while other potential function representations are not convergent always.

The alternatives to the potential function approach are as follows (I) State-Space approach: This method is essentially an expansion in a series in terms of the coefficient matrix of the field variables in ascending powers and applying Caley-Hamilton theorem, which requires extensive algebra, and (II) Eigenvalue approach: This method reduces the problem on vector-matrix differential equation to an algebraic eigenvalue problems and the solutions for the field variables are achieved by determining the eigenvalues and the corresponding eigenvectors of the coefficient matrix. In the eigenvalue approach the physical quantities are directly involved in the formulating of the problem and as such the boundary and initial conditions can be applied directly. Body forces and/or heat sources are also accommodated in both the theories, cf. Das et al. (1997; 2009), Lahiri et al. (2010), Kar and Lahiri (2004) and Sarkar and Lahiri (2012).

Saleh (2005), Youssef $(2006 ; 2009)$ studied some one-dimensional problems in thermoelasticity (generalized or coupled) including heat sources by the state space approach and generally in their models, heat sources included the Dirac delta function and Heavisides unit step function and consequently they obtained solutions easily by the state-space approach, but here we are interested in considering the heat source in any form and solve by the eigenvalue approach developed in Sarkar and Lahiri (2012).

In this work, we consider a one-dimensional problem for a half-space in the context of the L-S theory of generalized thermoelasticity with one relaxation time parameter subjected to a moving plane of heat source. The surface of the half-space is assumed to be traction free and subjected to the effects of a thermal shock. The main objective of this present paper is to study the above problem based on the LS theory with the help of the eigenvalue approach proposed by Sarkar and Lahiri (2012). See the Appendix for details. The inversion of the Laplace transform solutions are carried out numerically using Bellman method (Belman et al., 1966) and the obtained results are presented graphically. The effects of the moving heat source speed on temperature, stress and displacement are studied.

\section{Governing equations}

For a homogeneous, isotropic elastic solid, the basic equations for the linear generalized theory of thermoelasticity with one relaxation time parameter proposed by Lord and Shulman (1967) in the absence of body forces are:

(I) Equation of motion 


$$
\sigma_{i j, j}=\rho \frac{\partial^{2} u_{i}}{\partial t^{2}}
$$

(II) Heat conduction equation

$$
K T,{ }_{i i}=\left(\frac{\partial}{\partial t}+\tau \frac{\partial^{2}}{\partial t^{2}}\right)\left(\rho C_{E} T+\gamma T_{0} e\right)-\left(1+\tau \frac{\partial}{\partial t}\right) Q .
$$

(III) Stress-displacement-temperature relations

$$
\sigma_{\mathrm{ij}}=2 \mu e_{\mathrm{ij}}+\lambda e_{\mathrm{kk}} \delta_{\mathrm{ij}}-\gamma\left(T-T_{0}\right) \delta_{\mathrm{ij}}
$$

where $i, j=1,2,3$ refer to general coordinates.

\section{Formulation of the problem}

We consider a homogenous, isotropic and thermoelastic half-space which fills the region, $\Omega=\{x: 0 \leq x<\infty\}$ subjected to a moving plane of heat source on the bounding plane to the surface $x=0$. The body is initially at rest and the surface $x=0$ is assumed to be traction free. The governing equations will be written in the context of the theory of thermoelasticity with one relaxation time parameter (LS model), when the body has no body forces. Clearly, this is a one-dimensional problem and all the state functions depend only on the space variable $x$ and time variable $t$. The displacement components in this case are of the form

$$
u_{x}=u(x, t), \quad u_{y}=u_{z}=0 .
$$

We will use the Cartesian co-ordinates $(x, y, z)$ and the components of the displacement $u_{i}=(u, 0,0)$ and write them as follows

$$
\begin{aligned}
& \frac{\partial^{2} u}{\partial t^{2}}=(\lambda+2 \mu) \frac{\partial^{2} u}{\partial x^{2}}-\gamma \frac{\partial T}{\partial x} \\
& K \frac{\partial^{2} T}{\partial x^{2}}=\left(\frac{\partial}{\partial t}+\tau \frac{\partial^{2}}{\partial t^{2}}\right)\left(\rho C_{E} T+\gamma T_{0} e\right)-\left(1+\tau \frac{\partial}{\partial t}\right) Q \\
& \sigma=\sigma_{\mathrm{xx}}=(\lambda+2 \mu) \frac{\partial u}{\partial x}-\gamma\left(T-T_{0}\right)
\end{aligned}
$$

where $\quad e=\frac{\partial u}{\partial x}$.

To transform the above equations in non-dimensional forms, we define the following nondimensional variables 


$$
\begin{aligned}
& x^{*}=c_{1} \eta x, \quad u^{*}=c_{1} \eta u, \quad t^{*}=c^{2}{ }_{1} \eta t, \quad \tau^{*}=c^{2}{ }_{1} \eta \tau, \quad \Theta^{*}=\frac{\gamma\left(T-T_{0}\right)}{\lambda+2 \mu}, \\
& \sigma^{*}=\frac{\sigma}{(\lambda+2 \mu)}, \quad Q^{*}=\frac{\gamma Q}{K \rho \eta^{2} c_{1}^{4}}, \quad c_{1}^{2}=\frac{(\lambda+2 \mu)}{\rho}, \quad \eta=\frac{\rho C_{E}}{K} .
\end{aligned}
$$

Equations (3.1)-(3.3) then reduce to the following non-dimensional forms (the asterisks are dropped for convenience)

$$
\begin{aligned}
& \frac{\partial^{2} u}{\partial t^{2}}=\frac{\partial^{2} u}{\partial x^{2}}-\frac{\partial \Theta}{\partial x}, \\
& \frac{\partial^{2} \Theta}{\partial x^{2}}=\left(\frac{\partial}{\partial t}+\tau \frac{\partial^{2}}{\partial t^{2}}\right)\left(\Theta+\varepsilon \frac{\partial u}{\partial x}\right)-\left(1+\tau \frac{\partial}{\partial t}\right) Q \\
& \sigma=\frac{\partial u}{\partial x}-\Theta
\end{aligned}
$$

The space $x \geq 0$ is subjected to a moving plane of heat source of constant strength, releasing its energy continuously while moving along the positive direction of the $x$-axis with a constant velocity $v$. This moving heat source is assumed to be in the following non-dimensional form

$$
Q(x, t)=Q_{0} \delta(x-v t)
$$

where $Q_{0}$ is a constant of heat sources and $\delta($.$) is the Dirac delta function.$

\section{Formulation and solution of the vector-matrix differential equation}

Using the Laplace transform defined for any function $f(t)$ as follows

$$
\bar{f}(x, s)=\int_{0}^{\infty} f(x, t) e^{-s t} d t
$$

where $s$ is the transform parameter such that $\operatorname{Re}(s)>0$ and applying the above transform to both sides of Eqs (3.4)-(3.6) and assuming that all the initial state functions along with their derivatives with respect to $t$ are equal to zero, we obtain

$$
\begin{aligned}
& \frac{d^{2} \bar{u}}{d x^{2}}=s^{2} \bar{u}-\frac{d \Theta}{d x} \\
& \frac{d^{2} \bar{\Theta}}{d x^{2}}=\left(s+\tau s^{2}\right)\left(\bar{\Theta}+\varepsilon \frac{d \bar{u}}{d x}\right)-\frac{Q_{0}(1+\tau s)}{v} e^{-\frac{s}{v}},
\end{aligned}
$$




$$
\bar{\sigma}=\frac{d \bar{u}}{d x}-\bar{\Theta} .
$$

The resulting Eqs (4.1) and (4.2) can be written in the form of a vector-matrix differential equation as follows, see Sarkar and Lahiri (2012) and Appendix for details

$$
\frac{d V}{d x}=A(s) V(x, s)+f(x, s)
$$

where,

$$
\begin{aligned}
& V(x, s)=\left(\bar{\Theta}, \bar{u}, \frac{d \bar{\Theta}}{d x}, \frac{d \bar{u}}{d x}\right)^{T}, \\
& A(s)=\left(\begin{array}{cccc}
0 & 0 & 1 & 0 \\
0 & 0 & 0 & 1 \\
C_{31} & 0 & 0 & C_{34} \\
0 & s^{2} & 1 & 0
\end{array}\right), \\
& f=\left(0,0-\frac{Q_{0}(1+\tau s)}{v}, 0\right)^{T}
\end{aligned}
$$

and

$$
C_{31}=\left(s+\tau s^{2}\right), \quad C_{34}=\varepsilon\left(s+\tau s^{2}\right) .
$$

The eigenvectors $X_{i}$ of the coefficient matrix $A(s)$ corresponding to the eigenvalues $\lambda_{i}, i=1(l) 4$ of A $(x, s)$ respectively, may be found as

$$
X_{1}=(X)_{\lambda=\lambda_{1}}, \quad X_{2}=(X)_{\lambda=-\lambda_{1}}, \quad X_{3}=(X)_{\lambda=\lambda_{2}}, \quad X_{4}=(X)_{\lambda=-\lambda_{2}}
$$

where

$$
X=\left[s^{2}-\lambda^{2},-\lambda,-\lambda\left(s^{2}-\lambda^{2}\right),-\lambda\right]^{T} .
$$

Also, the eigenvalues of the coefficient matrix $\mathrm{A}(x, s)$ are of the form

$$
\lambda= \pm \lambda_{i}(i=1,2)
$$

where

$$
\lambda_{i}^{2}=\frac{\left(C_{31}+s^{2}+C_{34}\right)+(-1)^{i+1} \sqrt{\left(C_{31}+s^{2}+C_{34}\right)^{2}-4 s^{2} C_{31}}}{2}, \quad i=1,2 .
$$


Following the eigenvalue approach, see the Appendix for details, the field variables can be written as

$$
\begin{aligned}
& \bar{u}(x, s)=A_{1} \lambda_{1} e^{-\lambda_{1} x}+A_{2} \lambda_{2} e^{-\lambda_{2} x}+v \alpha e^{-\frac{s}{v} x}, \\
& \bar{\Theta}(x, s)=A_{1}\left(c_{42}-\lambda_{1}{ }^{2}\right) e^{-\lambda_{1} x}+A_{2}\left(c_{42}-\lambda_{2}{ }^{2}\right) e^{-\lambda_{2} x}+s \alpha\left(v^{2}-1\right) e^{-\frac{s}{v} x}
\end{aligned}
$$

where

$$
\alpha=\frac{Q_{0} s v(1+\tau s)}{\left(s^{2}-v^{2} \lambda_{1}^{2}\right)\left(s^{2}-v^{2} \lambda_{2}^{2}\right)} .
$$

Using Eqs (4.11) and (4.12) in Eq.(4.3), we obtain the stress component $\bar{\sigma}(x, s)$ as

$$
\bar{\sigma}(x, s)=-s^{2}\left(A_{1} e^{-\lambda_{1} x}+A_{2} e^{-\lambda_{2} x}\right)+s \alpha v^{2} e^{-\frac{s}{v} x}
$$

\section{Application}

\section{Unit step increase in temperature of the boundary of an elastic half-space.}

We consider a homogeneous, isotropic elastic solid occupying the half space $x \geq 0$. The initial conditions of the problem are assumed to be homogeneous. It is assumed that the boundary plane $x=0$ of the half space is subjected to a unit step increase in temperature which is traction free. Therefore the boundary conditions in the non-dimensional forms can be written as:

(a) mechanical boundary condition that the bounding plane to the surface $x=0$ has no traction anywhere, so we have

$$
\sigma(0, t)=0
$$

(b) the thermal boundary condition is

$$
\Theta(0, t)=\Theta_{0} \mathrm{H}(t)
$$

where $\Theta_{0}$ is the constant temperature and $\mathrm{H}(t)$ is the Heaviside unit step function. The transformed boundary conditions, for $t>0$ are

$$
\begin{aligned}
& \bar{\sigma}(0, s)=0, \\
& \bar{\Theta}(0, s)=\frac{\Theta_{0}}{s} .
\end{aligned}
$$


Using the boundary conditions Eq.(5.1) and Eq.(5.2) in Eqs (4.12) and (4.13), respectively, and after some simple computations, we get the constants $A_{1}, A_{2}$ in the following form

$$
\begin{aligned}
& A_{1}=\frac{\alpha s^{2}\left(2 v^{2}-1\right)-\Theta_{0}-\alpha v^{2} \lambda_{2}^{2}}{s\left(\lambda_{1}^{2}-\lambda_{2}^{2}\right)}, \\
& A_{2}=-\frac{\alpha s^{2}\left(2 v^{2}-1\right)-\Theta_{0}-\alpha v^{2} \lambda_{1}^{2}}{s\left(\lambda_{1}^{2}-\lambda_{2}^{2}\right)} .
\end{aligned}
$$

Equations (4.11)-(4.13) give the complete solution of the problem in the Laplace transform domain.

\section{Numerical example and discussions}

The Laplace inversion of the expressions given in Eqs (4.11)-(4.13) for displacement, temperature and stress respectively in the space-time domain are very complex and we prefer to develop an efficient computer programme for the inversion of these transforms. For this inversion of the Laplace transform, we follow the method of Bellman et al. (1966). The computations for the field variables are carried out for different values of $x$, three values of velocity, namely $v=0.1,0.2,0.3$ and for the time instants

$$
\begin{aligned}
& t_{1}=0.025775, \quad t_{2}=0.138382, \quad t_{3}=0.352509, \quad t_{4}=0.693147, \\
& t_{5}=1.21376, \quad t_{6}=2.04612, \quad t_{7}=3.67119,
\end{aligned}
$$

which are also the roots of the Legendre polynomial of degree seven, vide Bellman et al. (1966). A copper material is chosen for the purpose of numerical evaluations. The value of the non-dimensional constants $\varepsilon$ as well as the other constants are taken from Saleh (2005) as (in SI units)

$$
\varepsilon=0.0168, \quad \tau_{0}=0.05, \quad \Theta_{0}=5.0, \quad Q_{0}=10 .
$$

Using the cubic spline formulation the computed values of the field variables are then plotted in several graphs (see Figs 1-12) for different values of the space variable $x$ and for values of time $t$ taking three values of $v$. From Figs 1-12, we observe the following results:

(i) Figures 1-2 exhibit the variation of displacement against time $t$. Figure 1 shows that the displacement $u$ attains its maximum value near $t=t_{l}$ for $x=0.2$ and then decreases rapidly in $0 \leq t \leq 0.25$. Figure 2 shows that the displacement $u$ attains its minimum value near $t=t_{1}$ for $x=0.2$ and then increases rapidly in $0 \leq t \leq 0.27$ and then it shows no significant changes for $x \geq 0.28$. 


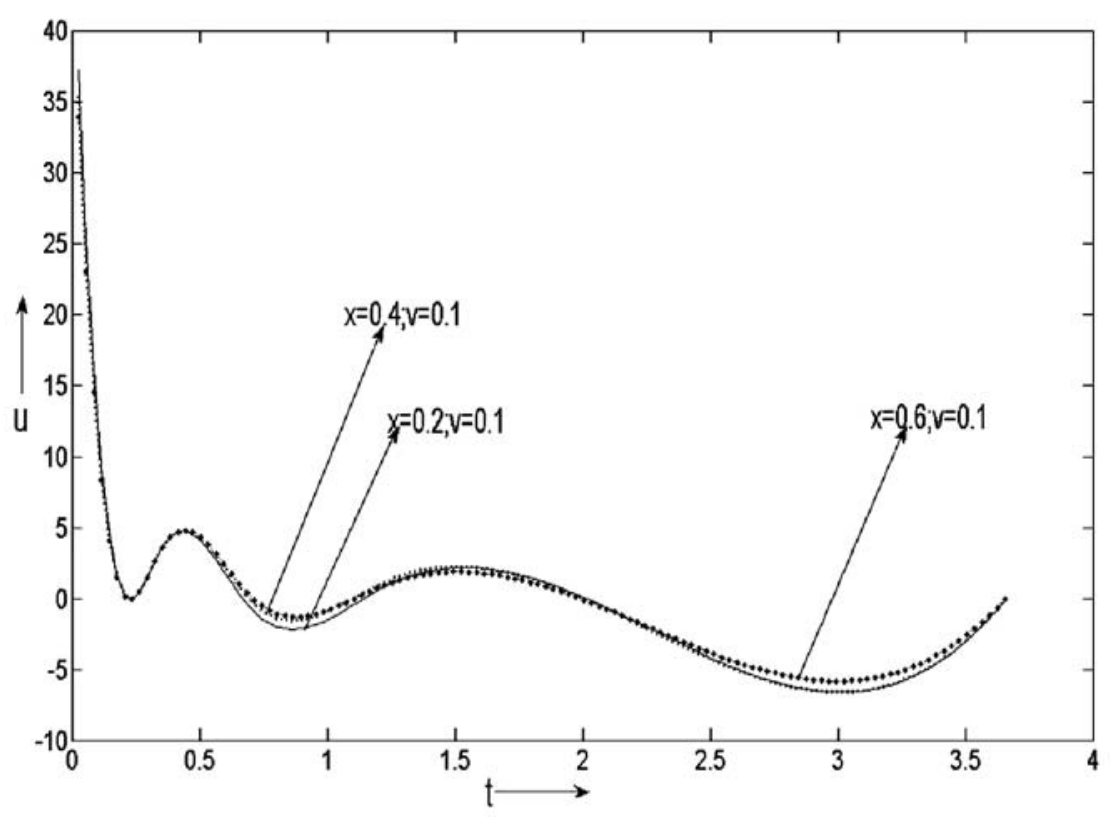

Fig.1. Displacement distribution against time $t$.

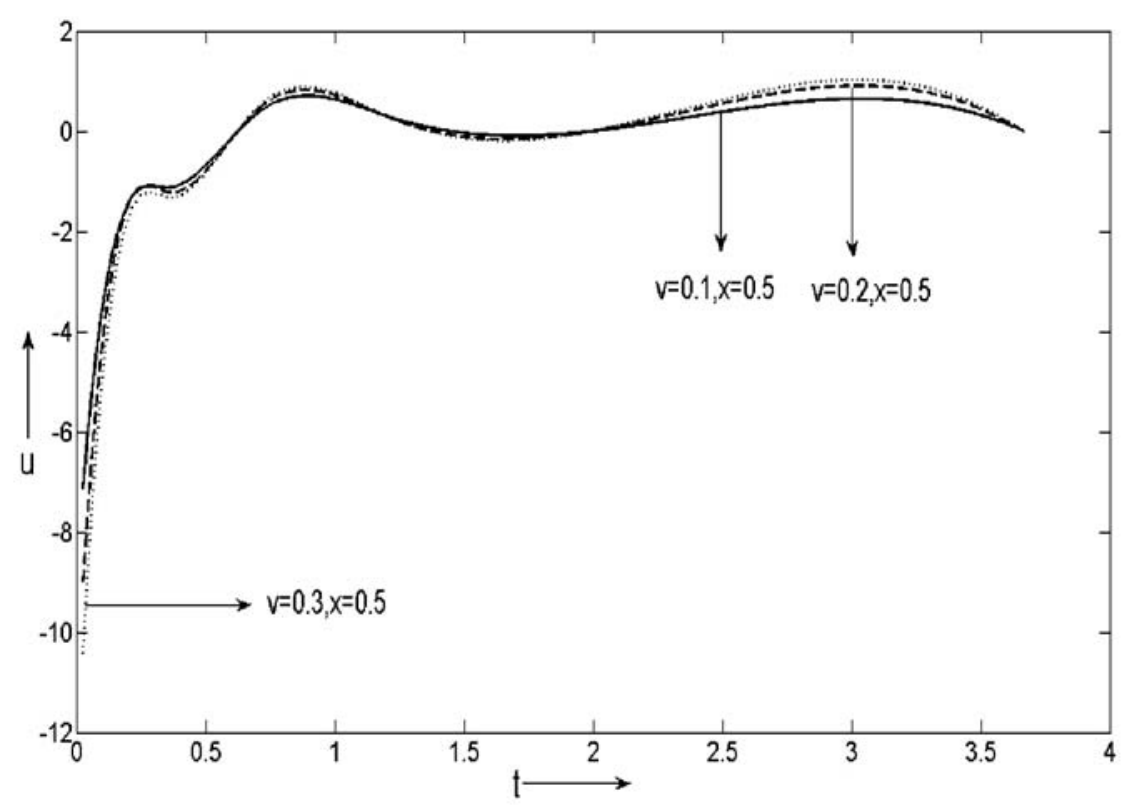

Fig.2. Displacement distribution against $t$.

(ii) Figures 3-4 exhibits the variation of temperature with time $t$ for different values of $x$ and $v$. In Fig.3, it is observed that the temperature $\Theta$ attains its maximum value near $t=t_{1}$ for $x=0.3$ and then decreases as time $t$ increases for fixed $x$ and $v$. The curves are smoother in the case $x=0.3$ than in the case $x=0.1,0.2$. From Fig.4, we see that the temperature $\Theta$ attains its maximum value near $t=t_{1}$ for $x=0.2$ and then decreases as time $t$ increases for fixed $x$ and $v$. In $0 \leq t \leq 1.2, \Theta$ increases as the velocity $v$ increases for fixed $x$ (see Fig.4). 


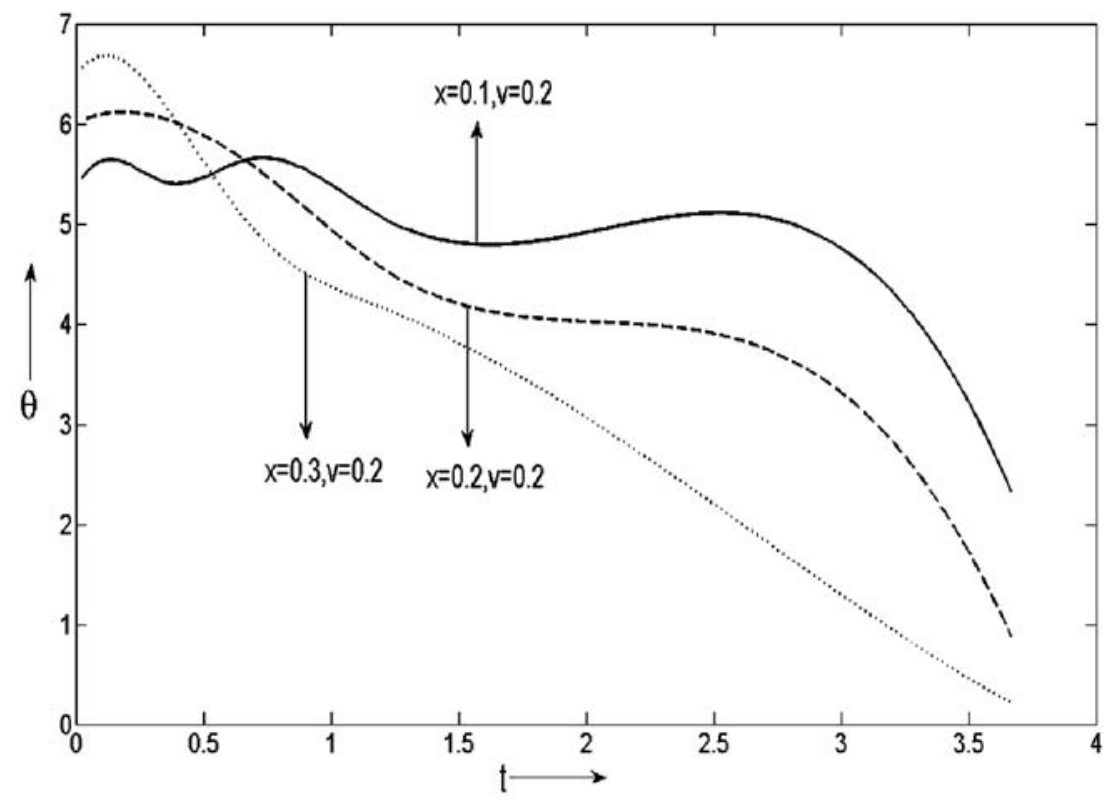

Fig.3. Temperature distribution against time $t$.

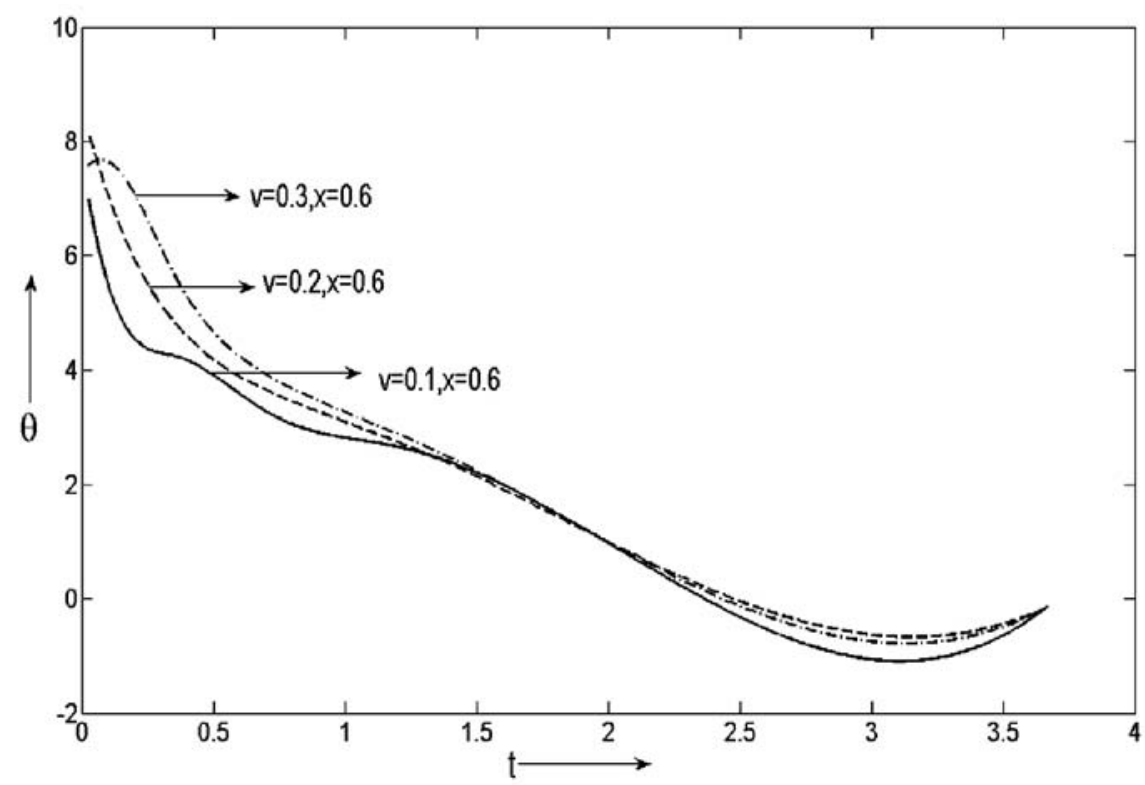

Fig.4. Temperature distribution against time $t$.

(iii) Figure 5 shows that there are no significant changes in $0 \leq t \leq 0.9$ and then the stress decreases as time $t$ increases and the numerical value of stress is maximum near $t=2.8$ for $x=0.2, v=0.3$. The curves are smoother in the case $x=0.3$ than in the case $x=0.1,0.2$. Again the absolute value of stress is maximum near $t=3.0$ for $x=0.6$ and $v=0.3$ (see Fig.6). 


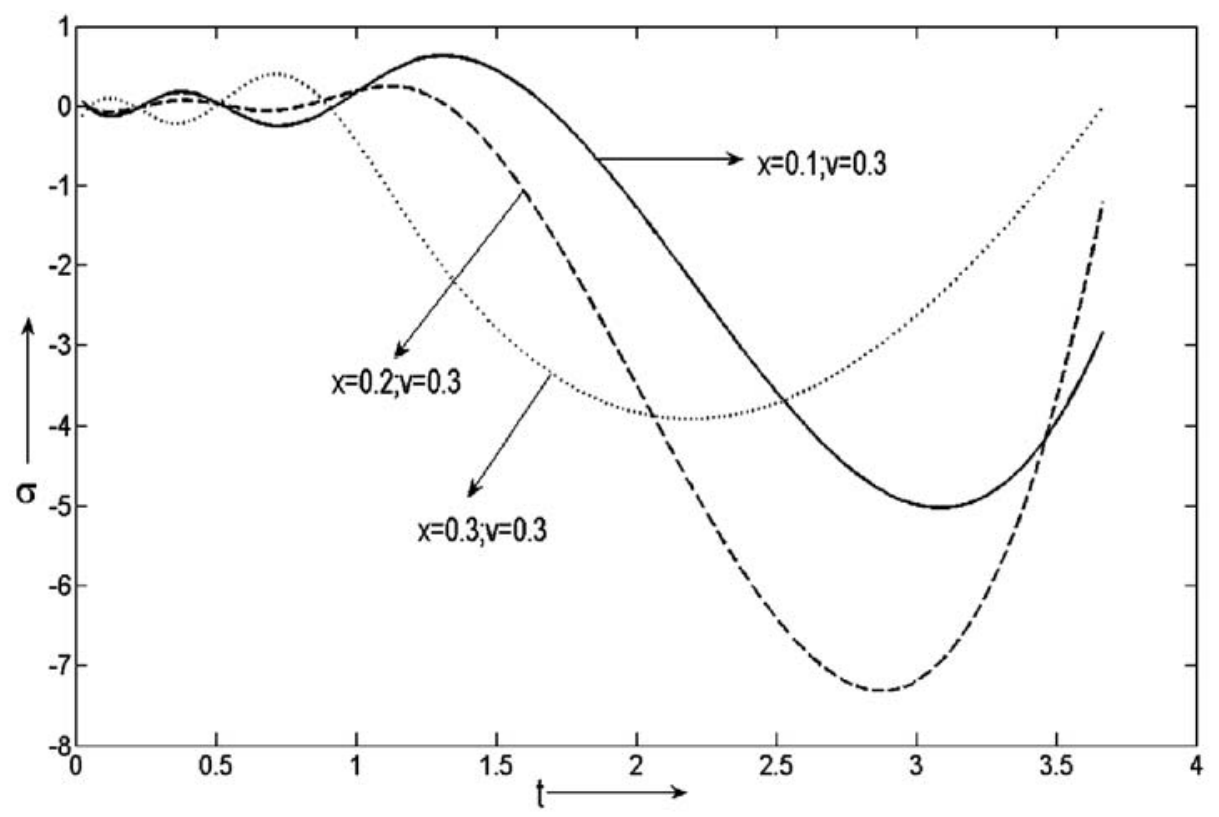

Fig.5. Stress distribution against time $t$.

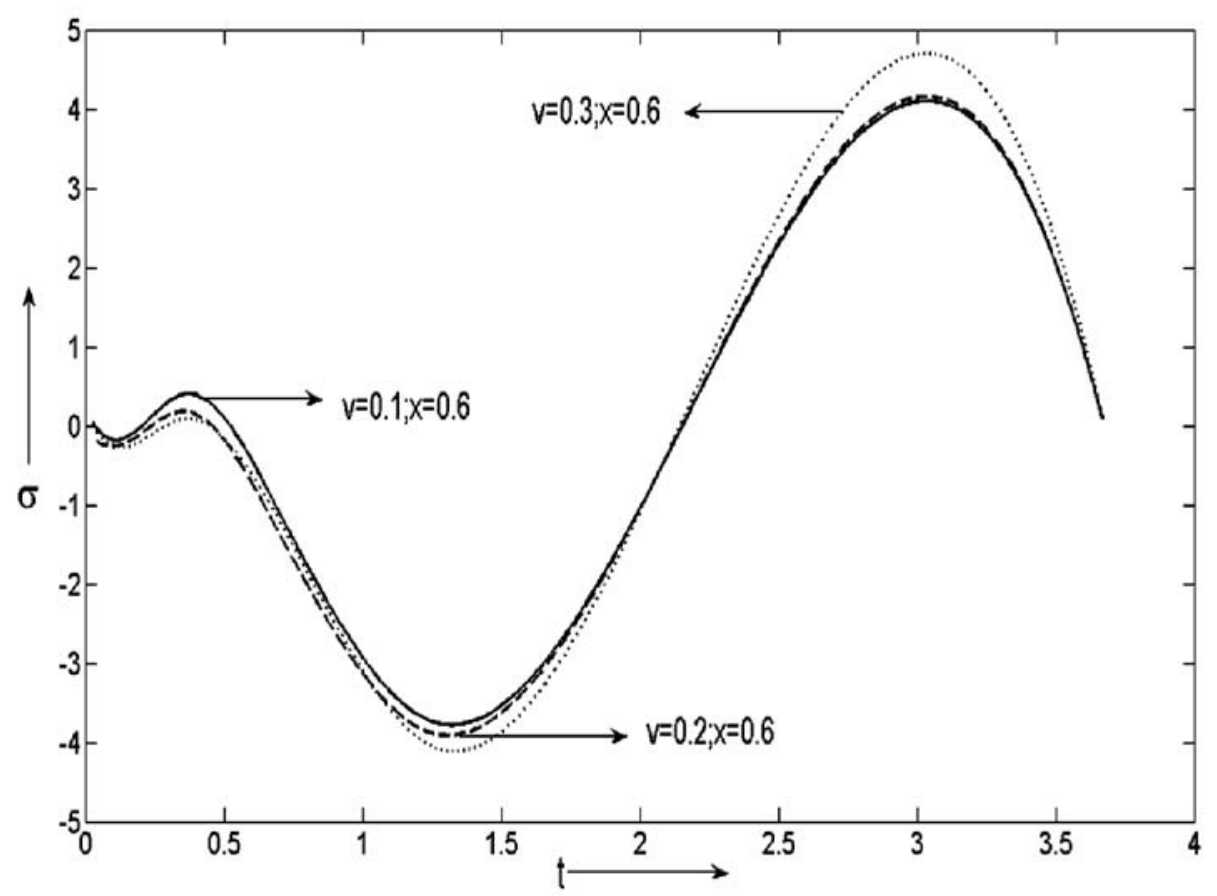

Fig.6. Stress distribution against time $t$.

(iv) Figure 7 shows that the numerical value of $u$ increases as the velocity $v$ increases for fixed; $t$ its absolute value is maximum near $x=0.1$ when $v=0.2$ and $t=t_{2}$. From Fig. 8 we see that the curves are smoother in the case $t=t_{3}$ than in the case $t=t_{4}, t_{5}$ for fixed value of $v$. In this case the numerical value of $u$ increases as time $t$ decreases when $x$ and $v$ are fixed. 


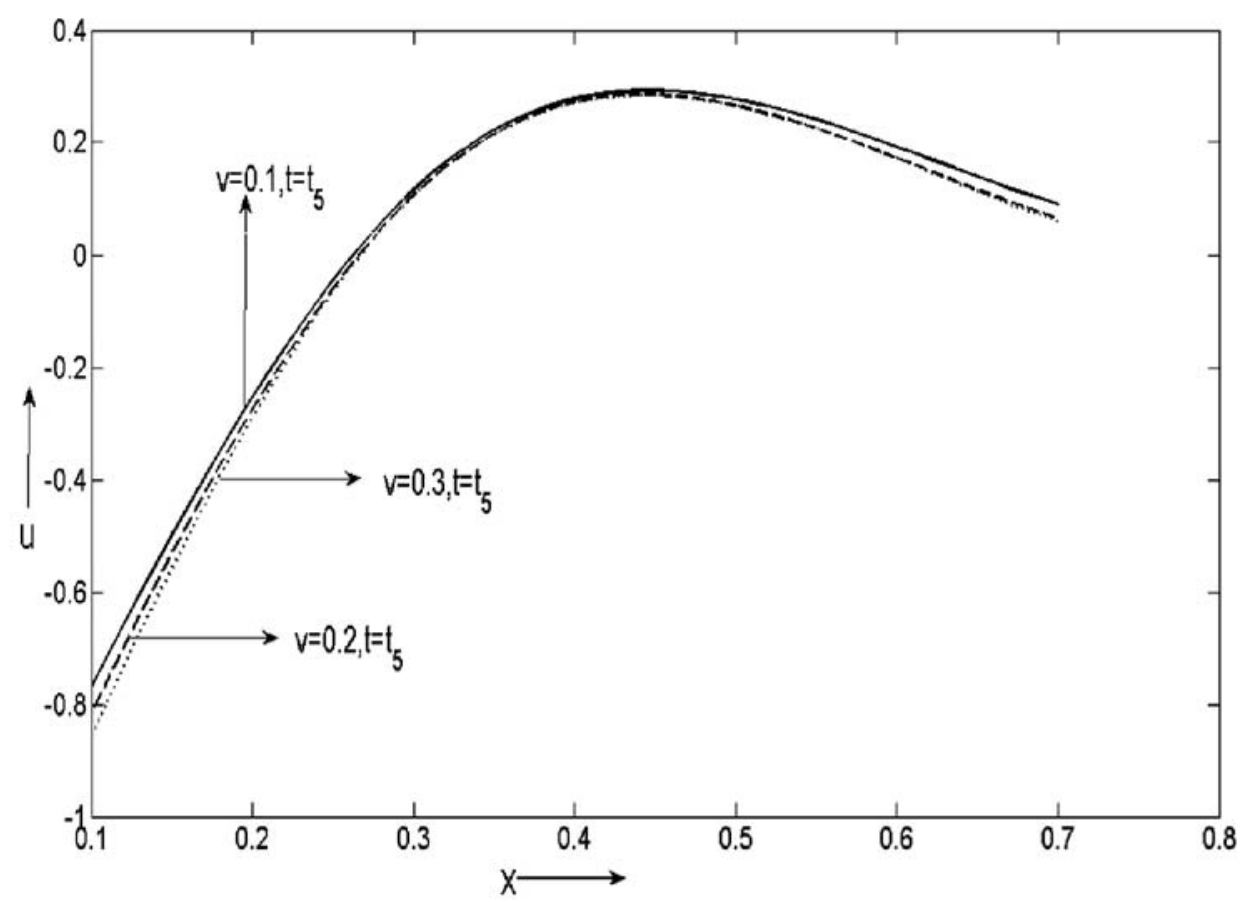

Fig.7. Displacement distribution against distance $x$.

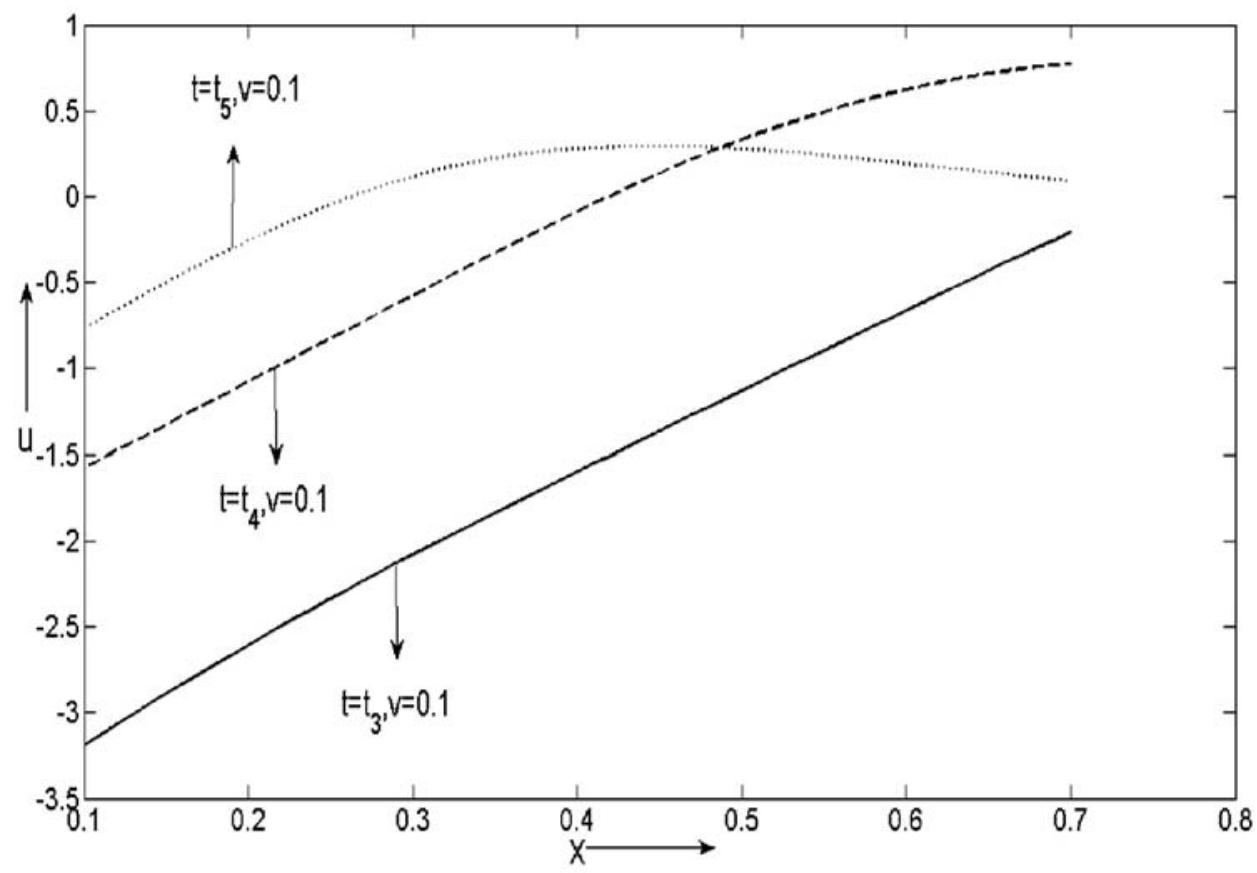

Fig.8. Displacement distribution against distance $x$.

(v) Figure 9 shows that the curves are smoother in the case $t=t_{5}$ than in the case $t=t_{6} t_{7}$. For fixed $x$ and $v, \Theta$ increases as time $t$ decreases and attains the maximum value near $x=0.1$ when $t=t_{6}$ and $v=0.2$ while in Fig. $10 \Theta$ increases as velocity $v$ increases for fixed $t$ and $x$ and finally it attains the maximum value at $x=0.1$ for $v=0.3$ and $t=t_{6}$. 


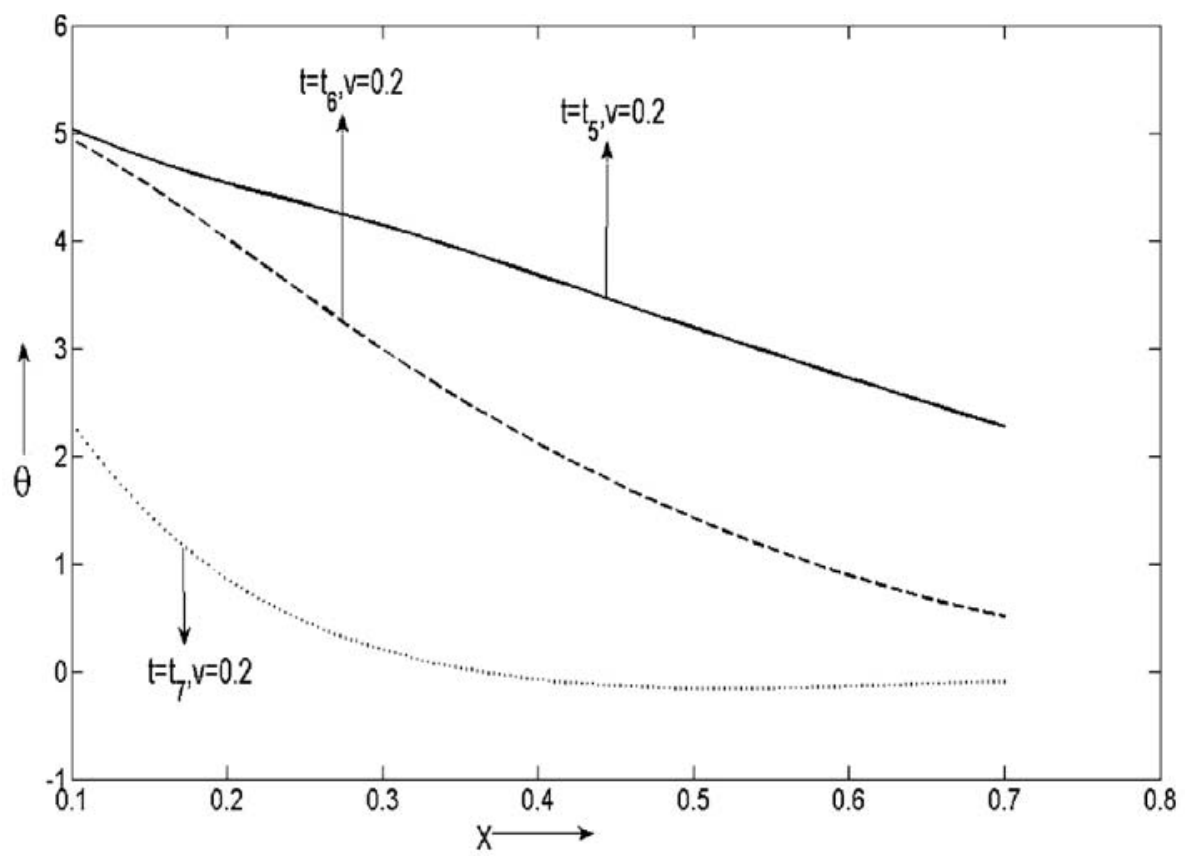

Fig.9. Temperature distribution against distance $x$.

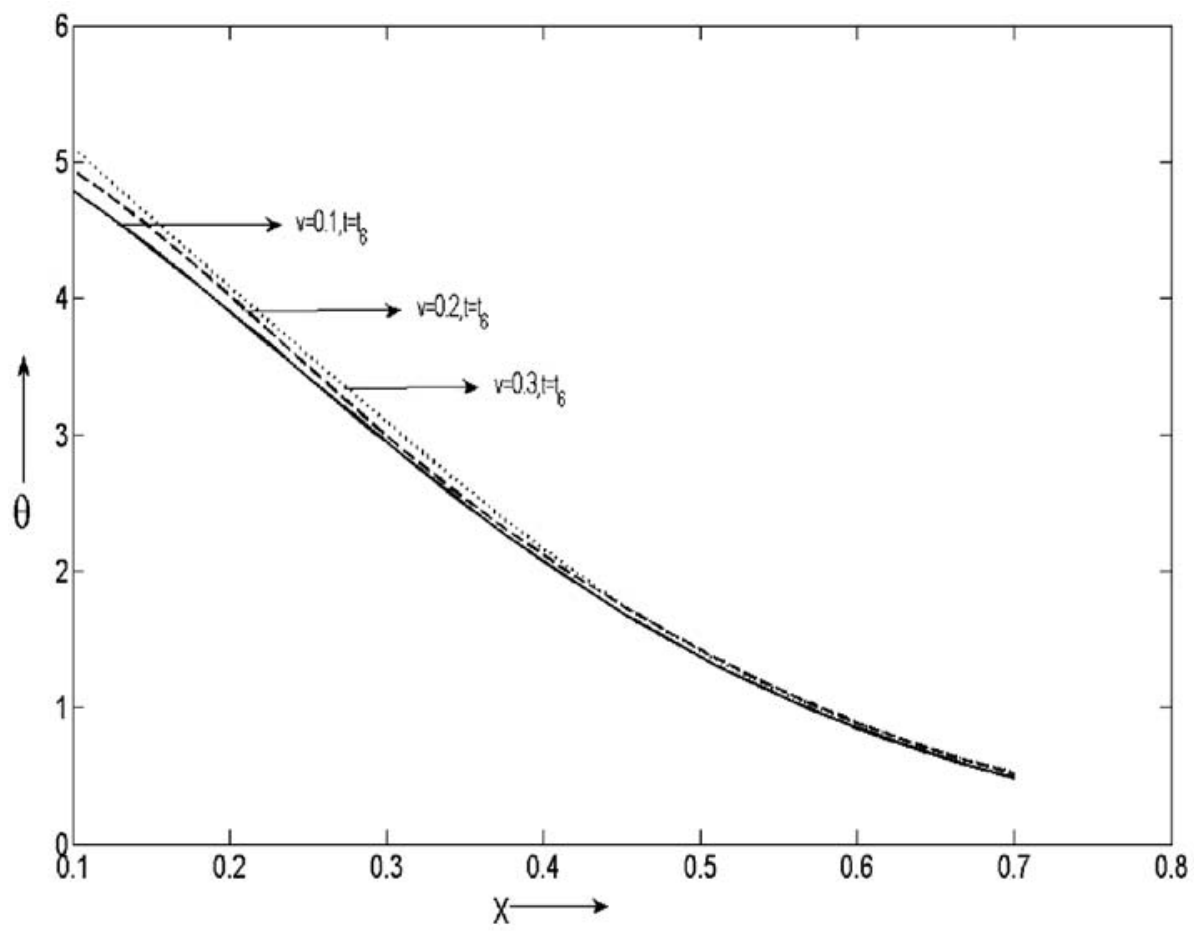

Fig.10. Temperature distribution against distance $x$.

(vi) Figures 11-12 exhibits the variation of stress with time $x$ for different values of $t$ and $v$. In Fig.11, we see that $\sigma$ attains its maximum value $x=0.1$ when $t=t_{7}$ and $v=0.2$. The curves are smoother in the case $t=t_{1}$ than in the case $t=t_{3}, t_{7}$. In Fig.12 stress $\sigma$ shows its compressive nature and in this case the numerical value of $\sigma$ increases as the velocity $v$ decreases. 


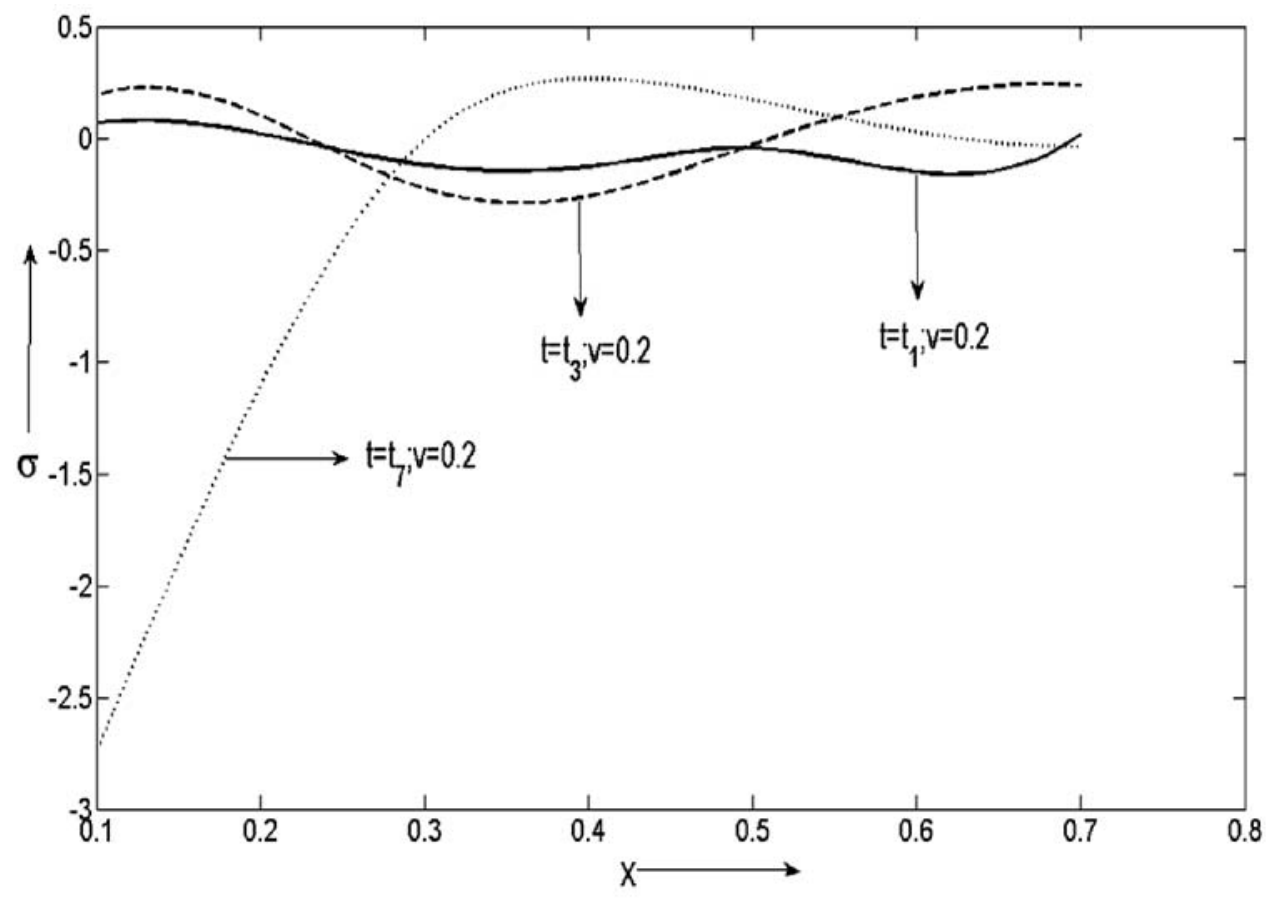

Fig.11. Stress distribution against distance $x$.

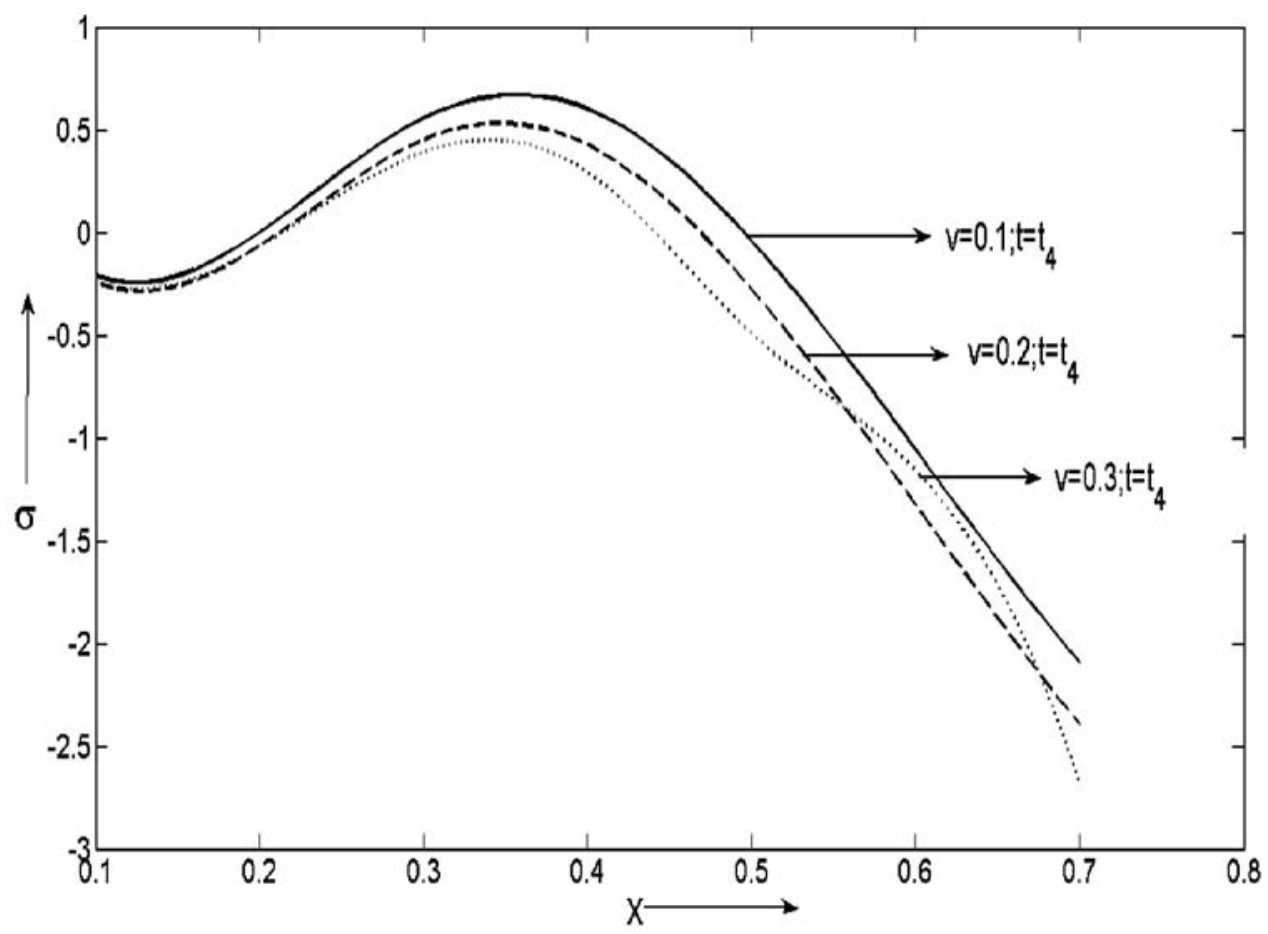

Fig.12. Stress distribution against distance $x$. 


\section{Concluding remarks}

We have investigated a one-dimensional dynamic response of a homogeneous isotropic and thermoelastic half-space subjected to a moving plane of heat sources in the context of the LS model of thermoelasticity. From the above discussions, we can arrive at the following conclusions:

(1) At a given instant, the non-zero values of non-dimensional temperature, displacement and stress are only in a bounded region.

(2) At $x=v t$, non-dimensional temperature attains its peak value once the time instant is given.

(3) The values of non-dimensional temperature, displacement and stress decrease as the moving heat source increases.

(4) The velocity of the heat sources has a significant role in all the field variables which are quite clear from Figs 6-9.

\section{Acknowledgements}

The authors are thankful to DST PURSE programme of the Department of Mathematics, Jadavpur University, Kolkata-700 032, India for the support of this work. Mr. Nantu Sarkar is grateful to the CSIR, New Delhi (India), for the award of a Junior Research Fellowship through the grant number 20-6/2008(II) EU-IV.

\section{Nomenclature}

$C_{P}$ - non-dimensional speeds of purely elastic dilatational wave

$C_{T}$-non-dimensional speeds of purely thermal wave

$c_{E}$ - specific heat at constant strain

$D-\equiv \frac{d}{d x}$

$e$ - cubical dilatation

$\mathrm{H}($.$) - Heaviside unit step function$

$K^{*}$ - material constant characteristic of the theory

$T$ - absolute temperature

$T_{0}$ - uniform reference temperature

$u_{i} \quad$ - displacement components $\left|\frac{T-T_{0}}{T_{0}}\right| \ll<1$

$\alpha_{T}$ - coefficient of volume expansion

$\gamma-=(3 \lambda+2 \mu) \alpha_{T}$

$\varepsilon-=\frac{\gamma^{2} T_{0}}{\rho C_{E}(\lambda+2 \mu)}$, thermoelastic coupling factor

$\lambda, \mu \quad$ - Lame' constants

$\rho-$ mass density

\section{References}

Anwar M. and Sherief H.H. (1988): State space approach to generalized thermoelasticity. - J. Thermal Stresses, vol.11, pp.353-365.

Belman R., Kalaba R.E. and Lockett J. (1966): Numarical inversion of the Laplace transform. - Amer Elsevier Publisher Co., New York.

Biot M. (1956): Thermoelasticity and irreversible thermodynamics. - J. Appl. Physics, vol.27, pp.240-253. 
Dhaliwal R.S. and Sherief H.H. (1980a): Generalized thermoelasticity for anisotropic media. - Quart. Appl. Math., vol.38 (1), pp.1-8.

Dhaliwal R.S. and Sherief H.H. (1980b): A uniqueness theorem and a variational principle for generalized thermoelasticity. - J. Thermal Stresses, vol.3, pp.223-230.

Das N.C., Lahiri A. and Giri R.R. (1997): Eigenvalue approach to generalized thermoelasticity. - Ind. J. Pure Appl. Math., vol.28, pp.1573-1594.

Das N.C., Lahiri A. and Sarkar S. (2009): Eigenvalue value approach three dimensional coupled thermoelasticity in a rotating transversly isotropic medium. - Tamsui Oxford J. Math. Sci., vol.25, pp.237-257.

Ezzat M.A., Othman M.I. and Smaan A.A. (2001): State space approach to two-dimensional electromagneticthermoelastic problem with two relaxation times. - Int. J. Eng. Sci., vol.39, pp.1383-1404.

Green A.E. and Lindsay K.A. (1972): Thermoelasticity. - J. Elasticity, vol.2, pp.1-7.

Green A.E. and Naghdi P.M. (1991): Are-examination of the basic postulate of thermo-mechanics. - Proc. Roy. Soc. London, vol.432, pp.171-194.

Green A.E. and Lindsay K.A. (1992): An unbounded heat wave in an elastic solid. - J. Thermal Stresses, vol.15, pp.253-264.

Green A.E. and Lindsay K.A. (1993): Thermoelasticity without energy dissipation.- J. Elasticity, vol.31, pp.189--208.

Ignaczak J. (1979): Uniqueness in generalized thermoelasticity. - J. Thermal Stresses, vol.2, pp.171-175.

Ignaczak J. (1982): A note on uniqueness in thermoelasticity with one relaxation time. - J. Thermal Stresses, vol. 5, pp. $257-263$.

Kar T.K. and Lahiri A. (2004): Eigenvalue approach to generalized thermoelasticity in an isotropic medium with an instantaneous heat sources. - Int. J. Appl. Mech. Eng., vol.9(1), pp.147-160.

Lord H.W. and Shulman Y.A. (1967): Generalized dynamical theory of thermoelasticity. - J. Mech. Physics of Solids, vol.15, pp.299-309.

Lahiri A., Das B. and Datta B. (2010a): Eigenvalue value approach to study the effect of rotation in three dimensional problem of generalized thermoelasticity. - Int. J. Appl. Mech. Eng., vol.15, pp.99-120.

Lahiri A., Das B. and Sarkar B. (2010b): Thermal stresses in an isotropic elastic slab due to prescribed surface temperature. - Adv. Theor. Appl. Mech., vol.3, pp.451-467.

Sherief H.H. (1987): On uniqueness and stability in generalized thermoelasticity. - Quart. Appl. Math., vol.5, pp.773778.

Sherief H.H. (1993): State space formulation for generalized thermoelasticity with one relaxation time including heat sources. - J. Thermal Stresses, vol.16, pp.163-180.

Sherief H.H. and Megahed F.A. (1999): A two-dimensional thermoelasticity problem for a half space subjected to heat sources. - Int. J. Solids Struc., vol.36, pp.1369-1382.

Sherief H.H. and Youssef H.M. (2004): Short time solution for a problem in magnetothermoelasticity with thermal relaxation. - J. Thernal Stresses, vol.27, pp.537-559.

Saleh H.A. (2005): Problem in generalized thermoelasticity for a half-space under the action of a body force. - J. Thermal Stresses, vol.28, pp.253-266.

Sarkar N. and Lahiri A. (2012): A three-dimensional thermoelastic problem for a half-space without energy dissipation. - Int. J. Eng. Sci., vol.51, pp.310-325.

Youssef H.M. (2006): Two-dimensional generalized thermoelasticity problem for a half-space subjected to ramp-type heating. - Eur. J. Mech., vol.25, pp.745-763.

Youssef H.M. (2009): Generalized thermoelastic infinite medium with cylindrical cavity subjected to moving heat source. - Mech. Res. Commun., vol.36, pp.487-496. 


\section{Appendix}

Let us consider a system of simultaneous differential equations in the form

$$
\frac{d \tilde{v}}{d x}=\tilde{A} \tilde{v}+\tilde{f}
$$

where

$$
\tilde{v}=\left(v_{1}, v_{2} \ldots \ldots . ., v_{n}\right)^{T}, \quad \tilde{A}=\left(a_{i j}\right)_{n \times n},
$$

and

$$
\tilde{f}=\left(f_{1,} f_{2}, \ldots \ldots \ldots \ldots, f_{n}\right)^{T}
$$

are real vectors and matrix. respectively and $f_{1}, f_{2}, f_{3}, \ldots, f_{n}$ are scaler functions of $x$.

Let us suppose that, the coefficient matrix $\tilde{A}$ can be written as

$$
\tilde{A}=V \Lambda V^{-1}
$$

where

and

$$
\Lambda=\left(\begin{array}{cccc}
\lambda_{1} & 0 & \ldots & 0 \\
0 & \lambda_{2} & \ldots & 0 \\
\ldots & \ldots & \ldots & \ldots \\
0 & 0 & \ldots & \lambda_{n}
\end{array}\right)
$$

$$
\tilde{V}=\left(V_{1}, V_{2}, \ldots \ldots \ldots \ldots, V_{n}\right)^{T}
$$

Here $\lambda_{1}, \lambda_{2}, \ldots, \lambda_{n}$ are the distinct eigenvalues of the coefficient matrix $\tilde{A}$ and $V_{1}, V_{2}, \ldots, V_{n}$ are the eigenvectors corresponding to the eigenvalues $\lambda_{1}, \lambda_{2}, \ldots, \lambda_{n}$, respectively. Now pre-multiplying Eq.(A.1) by $V^{-1}$ and using Eq.(A.2), we obtain

$$
\begin{aligned}
& V^{-1} \frac{d \tilde{v}}{d x}=V^{-1}\left(V \Lambda V^{-1}\right) \tilde{v}+V^{-1} \tilde{f}, \\
& \Rightarrow \frac{d\left(V^{-1} \tilde{v}\right)}{d x}=\Lambda\left(V^{-1} \tilde{v}\right)+V^{-1} \tilde{f}, \\
& \Rightarrow \frac{d \tilde{y}}{d x}=\Lambda \tilde{y}+V^{-1} \tilde{f}
\end{aligned}
$$

where, we define $\tilde{y}=V^{-1} \tilde{v} \Rightarrow \tilde{v}=V \tilde{y}$.

Now we solve the system of Eq.(A.3). Clearly, this equation represents a set of $n$-decoupled ordinary differential equations. A typical $r$-th equation of this system may be taken as 


$$
\frac{d y_{r}}{d x}=\lambda_{r} y_{r}+Q_{r}
$$

where,

$$
Q_{r}=\left(r \text {-th raw of } V^{-1}\right)(\tilde{f}) \text { and } V^{-1}=\left(w_{i j}\right) \text { (say). }
$$

Then $\quad Q_{r}=\sum_{i=1}^{n} w_{r i} f_{i}$.

The formal solution of Eq.(A.4) may be written as

$$
y_{r}=c_{r} e^{\lambda_{r} x}+e^{\lambda_{r} x} \int Q_{r} e^{-\lambda_{r} x} d x
$$

So

$$
\tilde{v}=V \tilde{y} \Rightarrow \tilde{v}=\sum_{r=1}^{n} V_{r} y_{r}
$$

which is the required solution of Eq.(A.1).

Received: January 14, 2012

Revised: April 30, 2013 